\title{
Pre-hospital i-gel blind intubation for trauma: a simulation study
}

\author{
Jae Guk Kim*, Wonhee Kim*, Gu Hyun Kang, Yong Soo Jang, \\ Hyun Young Choi, Hyeongtae Kim, Minji Kim
}

Department of Emergency Medicine, Kangnam Sacred Heart Hospital, Hallym University College of Medicine, Seoul, Korea

Objective This study aimed to evaluate the efficacy of i-gel blind intubation (IGI) as a rescue device for definitive airway management in ground intubation for pre-hospital trauma patients.

Methods A prospective randomized crossover study was conducted with 18 paramedics to examine intubation performance of two blind intubation techniques through a supraglottic airway devices (IGI and laryngeal mask airway Fastrach), compared with use of a Macintosh laryngoscope (MCL). Each intubation was conducted at two levels of patient positions (ground- and stretcher-level). Primary outcomes were the intubation time and the success rate for intubation.

Results The intubation time (sec) of each intubation technique was not significantly different between the two positions. In both patient positions, the intubation time of IGI was shortest among the three intubation techniques (17.9 \pm 5.2 at the ground-level and $16.9 \pm 3.8$ at the stretcher-level). In the analysis of cumulative success rate and intubation time, IGI was the fastest to reach 100\% success among the three intubation techniques regardless of patient position (all $\mathrm{P}<0.017$ ). The success of intubation was only affected by the intubation technique, and IGI achieved more success than MCL (odds ratio, 3.6; 95\% confidence interval, 1.1 to $11.6 ; P=0.03$ ).

Conclusion The patient position did not affect intubation performance. Additionally, the intubation time with blind intubation through supraglottic airway devices, especially with IGI, was significantly shorter than that with MCL.

Keywords Intubation; Airway management; Emergency medical technicians; Simulation training

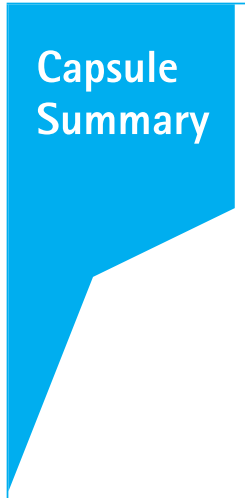

What is already known

Blind intubation using supraglottic airway devices such as the laryngeal mask airway Fastrach has also been used in pre-hospital trauma patients for achieving endotracheal intubation. There has been no study for the efficacy of i-gel blind intubation as a rescue device for definitive airway management.

\section{What is new in the current study}

Regardless of patient position, the intubation time of $i$-gel blind intubation was significantly shorter than that of laryngeal mask airway Fastrach and Macintosh laryngoscope. Therefore, i-gel blind intubation might be the appropriate option as a rescue device in pre-hospital traumatic patients.
Received: 26 October 2017

Revised: 11 December 2017

Accepted: 19 December 2017

Correspondence to: Gu Hyun Kang Department of Emergency Medicine, Kangnam Sacred Heart Hospital, Hallym University College of Medicine, 1 Singil-ro, Yeongdeungpo-gu, Seoul 07441, Korea

E-mail:drkang9@hanmail.net

*These two authors contributed equally to this study.

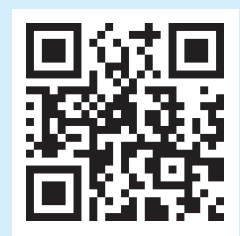

How to cite this article:

Kim JG, Kim W, Kang GH, Jang YS, Choi HY, Kim H, Kim M. Pre-hospital i-gel blind intubation for trauma: a simulation study. Clin Exp Emerg Med 2018;5(1):29-34.

This is an Open Access article distributed under the terms of the Creative Commons Attribution Non-Commercial License (http:// creativecommons.org/licenses/by-nc/4.0/). 


\section{INTRODUCTION}

Hypoxia and airway mismanagement contribute to up to $34 \%$ of pre-hospital deaths in trauma patients. ${ }^{1}$ Several studies have shown that $7 \%$ to $28 \%$ of patients with trauma require definitive airway management, including endotracheal intubation (ETI)., ${ }^{2,3}$ Moreover, paramedics mostly perform ground intubation in the pre-hospital setting for definitive airway management. However, during ground intubation using a Macintosh laryngoscope ( $\mathrm{MCL}_{\text {; }}$ GreenLite, Truphatek International, Netanya, Israel), it is not easy to expose the vocal cords, compared with a sternum-level bed setting. ${ }^{4}$ Moreover, cervical immobilization of trauma patients makes it more difficult to visualize the vocal cords.

Video laryngoscopes (VL) have been tried to resolve hypoxia in pre-hospital trauma patients. The use of VL has advantages for securing a good view of the glottis in cervical-immobilized patients. Nevertheless, paramedics using VL encountered difficult circumstances in which the screen went black due to the camera of the VL being covered with blood or vomitus of patients. ${ }^{5}$

Blind intubation using the laryngeal mask airway (LMA) Fastrach (LMF; Teleflex, Athlone, Ireland) has also been used in trauma patients for achieving ETI. It requires minimal head and neck movement while placing them into the patient's oropharynx, and facilitates ETI as the patient is being simultaneously ventilated. ${ }^{6}$ In previous studies using a sternum-level bed setting, LMF had a longer intubation time compared with $\mathrm{MCL}_{1}^{7}$ but equivalent performance to i-gel blind intubation (IGI) regarding intubation time and success rate. ${ }^{8}$ There has been no comparison study for $\mid \mathrm{GI}$, LMF and MCL in the ground position.

Thus, we evaluated the efficacy of IGI as a rescue device for definitive airway management, compared with LMF and MCL in ground intubation for pre-hospital trauma patients. We hypothesized that IGI would have better intubation performance compared with LMF and MCL regarding intubation time and success rate.

\section{METHODS}

\section{Study design}

We conducted a randomized crossover manikin study to examine intubation performance using IGI, LMF and MCL at two different patient positions (ground- and stretcher-level). This study was performed at our hospital in June 2016. The local ethics committee approved this study and a waiver for participants was obtained in April 2016 (institutional review board no. 2016-03-27). We registered the study protocol in CRIS (Clinical Research Information Service, KCT0001942) in June 2016 before study initiation.

\section{Equipment and material}

At each patient position, participants attempted ETI using three types of intubation techniques: direct laryngoscopy using $\mathrm{MCL}$ and blind intubation with two different supraglottic airway devices (SADs). The MCL had a size-4 curved blade, and direct laryngoscopy was performed using with a Satin Slip Stylet (Mallinckrodt Medical, St. Louis, MO, USA). Two types of SADs were used for blind intubation in this study: a size-4 i-gel (Intersurgical, Berkshire, UK) and a size-4 LMF. A 7.0-mm internal diameter endotracheal tube (ETT) (Mallinckrodt Hi-Lo Oral/Nasal Tracheal Tube Cuffed Murphy Eye; Covidien, Dublin, Ireland) was used for direct laryngoscopy, and a 6.0-mm internal diameter ETT of the LMF was used for blind intubation through SADs. Each ETT was lubricated by airway lubricant (Airway lubricant glycerol version; Laerdal Medical, Stavanger, Norway) before performing ETI.

We used an airway trainer manikin (AirSim Advance Combo Bronchi; TruCorp, Belfast, Northern Ireland) to perform ETI. The simulation airway setting was regulated by cervical immobilization using a cervical collar (Perfit ACE; Ambu, Ballerup, Denmark). The stretcher-level patient position was simulated using a $91-\mathrm{cm}$-height of an ambulance stretcher (stretcher, 189 $\times 58 \times 91$ cm; Sungwoo Motors, Eumseong, South Korea).

\section{Participants}

The sample size was calculated based on a previous study regarding the time required for ETI.? The intubation times (mean \pm standard deviation) in that study were as follows: IGI $(17.2 \pm 7.7 \mathrm{sec}-$ onds) and LMF (26.3 \pm 13.6 seconds). To detect a $34 \%$ difference in intubation time with a power of 0.8 , at least 16 operators were needed. We estimated that 20 operators would be adequate, considering an estimated $20 \%$ dropout rate.

We recruited healthy volunteer paramedics who were between 18 and 60 years of age and in charge of rescue departments. Any persons having cardiovascular, neurological, or musculoskeletal diseases affecting any part of the body and pregnant women were excluded.

\section{Interventions}

All participants completed a brief questionnaire regarding demographic information (age, sex, body weight, and height) and prior clinical ETI experience with MCL, IGI, and LMF (Table 1). Before starting the experiments, instructors gave lectures, approximately 1 hour in duration, about ETI and SADs. Participants had a 10minute ETI practice time to familiarize themselves with all devices.

Eighteen participants were finally enrolled, and they were randomly allocated into two groups: group $A(n=9)$ and group $B$ $(n=9)$ (Fig. 1). After group allocation, the participants performed 
Table 1. Baseline characteristics

\begin{tabular}{lc}
\hline Characteristics & Value $(\mathrm{n}=17)$ \\
\hline Sex, male & $10(58.8)$ \\
Age $(\mathrm{yr})$ & $31.5 \pm 5.9$ \\
Height $(\mathrm{cm})$ & $169.7 \pm 8.4$ \\
Weight $(\mathrm{kg})$ & $64.3 \pm 10.6$ \\
Body mass index $\left(\mathrm{kg} / \mathrm{m}^{2}\right)$ & $22.2 \pm 2.5$ \\
Participants & \\
$\quad$ 1st level EMT & $17(100)$ \\
Paramedic experience $(\mathrm{mo})$ & $58.8 \pm 66.8$ \\
Intubation experiences & $12(70.6)$ \\
Intubation experience (times) & \\
MCL & $5.9 \pm 8.7$ \\
IGI & $\mathrm{ND}$ \\
LMF & $\mathrm{ND}$ \\
\hline
\end{tabular}

Categorical variables are given as numbers (percentage). Continuous variables are given as mean \pm standard deviation.

EMT, emergency medical technician; MCL, Macintosh laryngoscope; IGI, i-gel blind intubation; LMF, laryngeal mask airway Fastrach; ND, not done.

ETls in a random order generated by a sequence generator (http:// www.random.org) to minimize the learning effects for ETI. Only one ETI attempt was allowed for each device at phase 1 or 2. In phase 1, each participant of group A attempted ETls using MCL or IGI or LMF according to the randomly given order at the groundlevel position. Similarly, the participants of group B $(n=9)$ attempted ETls at the stretcher-level position. After a mandatory rest period of 30 minutes, the participants of each group performed ETls at the other level patient position in phase 2 .

\section{Outcomes}

The primary outcomes were the intubation time and the success rate of ETI. The time required for SAD insertion (device insertion time, DT) was defined as the time from picking up SADs to complete insertion into the manikin. For MCL, DT was alternatively defined as the time from picking up the MCL to complete exposure of the larynx. The time required for ETT insertion (tube insertion time, T) was defined as the time from picking up the SADs or MCL to successful insertion of the ETT. The time for first ventilation (FVT) was defined as the time from picking up SADs or MCL to successful first ventilation. Successful ETI was defined as a visible chest rise by bagging with bag valve mask. Intubation failure was defined as follows: esophageal ETI, incomplete progression of $E T$ in IGI or LMF, or exceeding the time limit of 120 seconds. ${ }^{9}$ The glottis view was measured according to the Cormack and Lehane grade.

\section{Statistical analysis}

The data were compiled using a standard spreadsheet application (Excel; Microsoft, Redmond, WA, USA) and analyzed using the
Assessed for eligiblity $(n=18)$

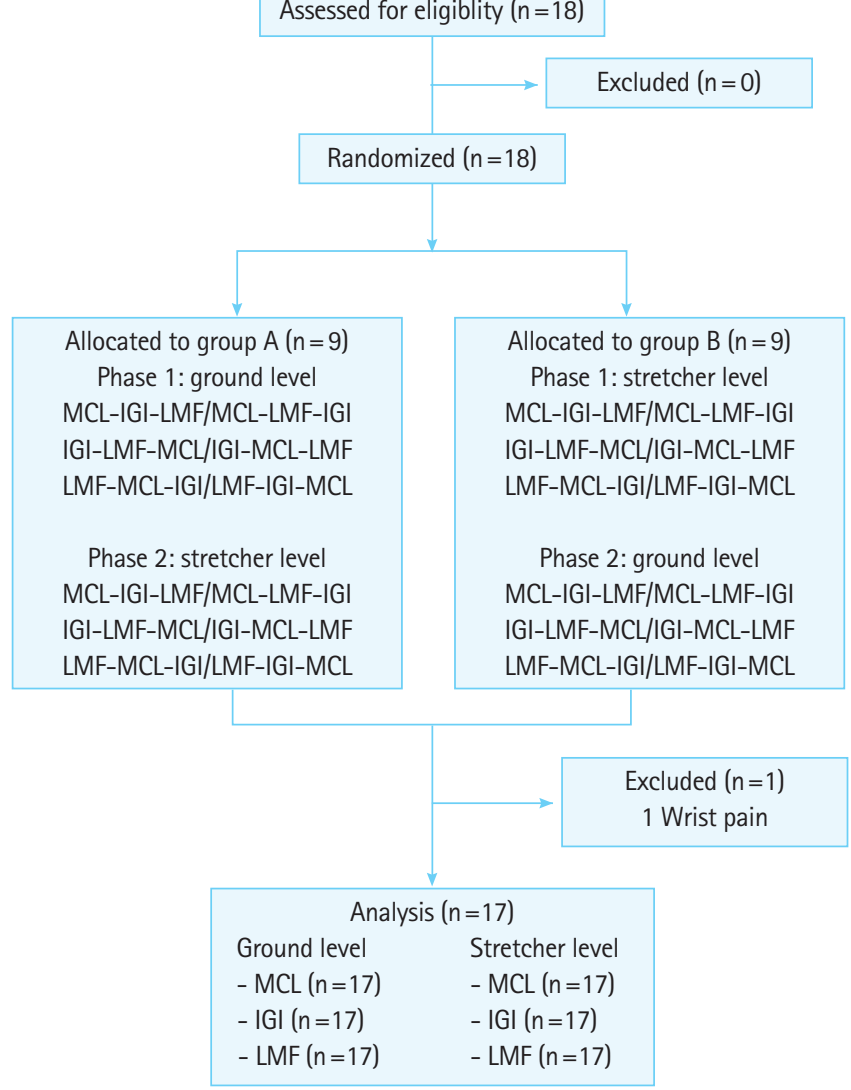

Fig. 1. Flow diagram of research process. A participant was exlcuded during the study due to complaint of wrist pain. MCL, Macintosh laryngoscope; IGI, i-gel blind intubation; LMF, laryngeal mask airway Fastrach

IBM SPSS Statistics ver. 20.0 (IBM Corp., Armonk, NY, USA). We generated descriptive statistics and presented them as frequencies and percentages for categorical data and mean \pm standard deviation for continuous data. To compare intubation times among the three intubation techniques, the Friedman test (nonparametric data) was used for continuous variables. A post-hoc analysis was conducted with the Wilcoxon signed rank test (nonparametric data) or a paired t-test (parametric data) using the Bonferroni correction. The McNemar test or Cochran's 0 test were used to compare categorical variables, such as the success rate for ETI and glottis view. Kaplan-Meier analysis was performed to analyze the cumulative success rate regarding intubation time. A post-hoc analysis was also performed using the logrank test with the Bonferroni correction. Additionally, multivariate logistic regression analysis was conducted to analyze the factors influencing successful ETI. For all analyzed data, $\mathrm{P}<0.05$ was considered statistically significant. In contrast, in post-hoc analysis, $\mathrm{P}<0.017$ was considered significant. 


\section{RESULTS}

\section{Baseline characteristics}

Eighteen participants were enrolled in this study, and one participant was excluded. The baseline characteristics of the participants are shown in Table 1.

\section{Intubation performance at two different positions}

The intubation time was not significantly different between the two different positions regardless of the type of intubation technique (Table 2). The DT was not significantly different among the three intubation techniques in the ground-level position $(\mathrm{P}=$
0.10). At the stretcher-level position, the DT of IGI was significantly shorter than that of $\operatorname{LMF}(6.2 \pm 2.4$ vs. $10.7 \pm 3.7, P=0.01)$

The $\Pi$ of IGI was significantly shorter than that of LMF in the ground-level position ( $13.8 \pm 4.5$ vs. $20.4 \pm 5.1, P=0.005)$. At the stretcher-level position, the $\Pi$ of IGI was the shortest among the three intubation techniques $(P=0.005)$, although those of $\mathrm{MCL}$ and LMF were not significantly different.

For the comparison of FVT, the use of IGI reduced FVT compared with LMF in the ground-level position (17.9 \pm 5.2 vs. $24.7 \pm 6.4, P=0.005$ ). At the stretcher-level position, the FVT of IGI was the shortest among the three intubation techniques, although those of MCL and LMF were not significantly different

Table 2. Comparison of intubation performance between two patient positions

\begin{tabular}{|c|c|c|c|c|c|c|c|c|c|c|}
\hline & & \multicolumn{3}{|c|}{$\mathrm{MCL}$} & \multicolumn{3}{|c|}{ LMF } & \multicolumn{3}{|c|}{$|G|$} \\
\hline & & Ground-level & Stretcher-level & P-value & Ground-level & Stretcher-level & P-value & Ground-level & Stretcher-level & P-value \\
\hline \multirow[t]{3}{*}{ Intubation time (sec) } & $\mathrm{DT}^{\mathrm{a}}$ & $19.5 \pm 16.1$ & $10.2 \pm 4.6$ & 0.22 & $11.4 \pm 2.6$ & $10.7 \pm 3.7$ & $0.93^{b)}$ & $6.6 \pm 2.8$ & $6.2 \pm 2.4$ & 0.93 \\
\hline & $\Pi$ & $33.1 \pm 23.7$ & $21.7 \pm 13.7$ & 0.22 & $20.4 \pm 5.1$ & $22.8 \pm 16.3$ & 0.92 & $13.8 \pm 4.5$ & $13.2 \pm 4.0$ & $0.92^{b)}$ \\
\hline & $\mathrm{FVT}$ & $39.0 \pm 23.8$ & $28.8 \pm 14.2$ & 0.22 & $24.7 \pm 6.4$ & $27.3 \pm 16.6$ & 0.37 & $17.9 \pm 5.2$ & $16.9 \pm 3.8$ & 0.87 \\
\hline \multirow[t]{2}{*}{ Success rate } & Success & $8(47.1)$ & $12(70.6)$ & 0.58 & $14(82.4)$ & $12(70.6)$ & 0.06 & $13(76.5)$ & 15 (88.2) & 0.007 \\
\hline & Fail & $9(52.9)$ & $5(29.4)$ & & $3(17.6)$ & $5(29.4)$ & & $4(23.5)$ & $2(11.8)$ & \\
\hline \multirow[t]{2}{*}{$\mathrm{CL}$ grade } & I-II & $8(47.1)$ & $11(64.7)$ & 0.79 & NA & NA & NA & NA & NA & NA \\
\hline & III-IV & $6(35.3)$ & $6(35.3)$ & & NA & NA & & NA & NA & \\
\hline
\end{tabular}

Continuous variables are given as mean \pm standard deviation. Categorical variables are given as numbers (percentage). P-value for intubation time was calculated by Wilcoxon signed rank test. P-value for success rate and CL grade was calculated by McNemar's test.

MCL, Macintosh laryngoscope; LMF, laryngeal mask airway Fastrach; IGI, i-gel blind intubation; DT, device insertion time; $\Pi$, tube insertion time; FVT, first ventilation time; $\mathrm{CL}$ grade, Cormack and Lehane grade; NA, not available.

${ }^{\text {a) }}$ Vocal cords exposure time in MCL. ${ }^{\text {b) }}$ Calculated by paired t-test.

Table 3. Comparison of intubation performance using three intubation techniques at each patient position

\begin{tabular}{|c|c|c|c|c|c|c|c|c|c|}
\hline \multirow{3}{*}{$\begin{array}{l}\text { Patient position } \\
\text { Ground }\end{array}$} & \multirow{2}{*}{\multicolumn{2}{|c|}{ Intubation performance }} & \multirow{2}{*}{$\mathrm{MCL}$} & \multirow{2}{*}{ LMF } & \multirow{2}{*}{$|G|$} & \multirow{2}{*}{ P-value ${ }^{a)}$} & \multicolumn{3}{|c|}{ P-value ${ }^{b)}$} \\
\hline & & & & & & & MCL vs. LMF & LMF vs. IGI & IGI vs. MCL \\
\hline & Intubation time (sec) & $\mathrm{DT}^{\mathrm{c})}$ & $19.5 \pm 16.1$ & $11.4 \pm 2.6$ & $6.6 \pm 2.8$ & 0.105 & 0.345 & 0.028 & 0.025 \\
\hline & & $\Pi$ & $33.1 \pm 23.7$ & $20.4 \pm 5.1$ & $13.8 \pm 4.5$ & 0.039 & 0.173 & $0.005^{\mathrm{d})}$ & 0.026 \\
\hline & & FVT & $39.0 \pm 23.8$ & $24.7 \pm 6.4$ & $17.9 \pm 5.2$ & 0.018 & 0.075 & $0.005^{\mathrm{d})}$ & 0.022 \\
\hline & Success rate & Success & $8(47.1)$ & $14(82.4)$ & $13(76.5)$ & 0.092 & 0.109 & 1.000 & 0.180 \\
\hline & & Fail & $9(52.9)$ & $3(17.6)$ & $4(23.5)$ & & & & \\
\hline & $\mathrm{CL}$ grade & $|-| \mid$ & $8(47.1)$ & NA & NA & NA & NA & NA & NA \\
\hline & & III-IV & $6(35.3)$ & NA & NA & & & & \\
\hline Stretcher & Intubation time (sec) & DT & $10.2 \pm 4.6$ & $10.7 \pm 3.7$ & $6.2 \pm 2.4$ & 0.013 & 0.374 & $0.010^{d)}$ & 0.020 \\
\hline & & $\Pi$ & $21.7 \pm 13.7$ & $22.8 \pm 16.3$ & $13.2 \pm 4.0$ & 0.013 & 0.173 & $0.010^{d)}$ & $0.004^{d)}$ \\
\hline & & FVT & $28.8 \pm 14.2$ & $27.3 \pm 16.6$ & $16.9 \pm 3.8$ & 0.016 & 0.374 & $<0.001^{\mathrm{d})}$ & $0.006^{d)}$ \\
\hline & Success rate & Success & $12(70.6)$ & 12 (70.6) & 15 (88.2) & 0.325 & 1.000 & 0.375 & 0.375 \\
\hline & & Fail & $5(29.4)$ & 5 (29.4) & $2(11.8)$ & & & & \\
\hline & $\mathrm{CL}$ grade & |- I| & $11(64.7)$ & NA & NA & NA & NA & NA & NA \\
\hline & & III-IV & $6(35.3)$ & NA & NA & & & & \\
\hline
\end{tabular}

Continuous variables are given as mean \pm standard deviation. Categorical variables are given as numbers (percentage).

MCL, Macintosh laryngoscope; LMF, laryngeal mask airway Fastrach; IGI, i-gel blind intubation; DT, device insertion time; $\Pi$, tube insertion time; FVT, first ventilation time; CL grade, Cormack and Lehane grade; NA, not available.

${ }^{a)}$ Calculated by Friedman test for continuous variables and Cochrane's $\mathrm{Q}$ test for categorical variables. $\mathrm{P}<0.05$ was considered significant. ${ }^{\text {b) }} \mathrm{Calculated}$ by Wilcoxon signed rank test for continuous variables and McNemar's test for categorical variables in post hoc analysis. ${ }^{c}$ Vocal cords exposure time in $\mathrm{MCL}$. ${ }^{\mathrm{d}} \mathrm{P}<0.017$ was considered significant using Bonferroni correction. 

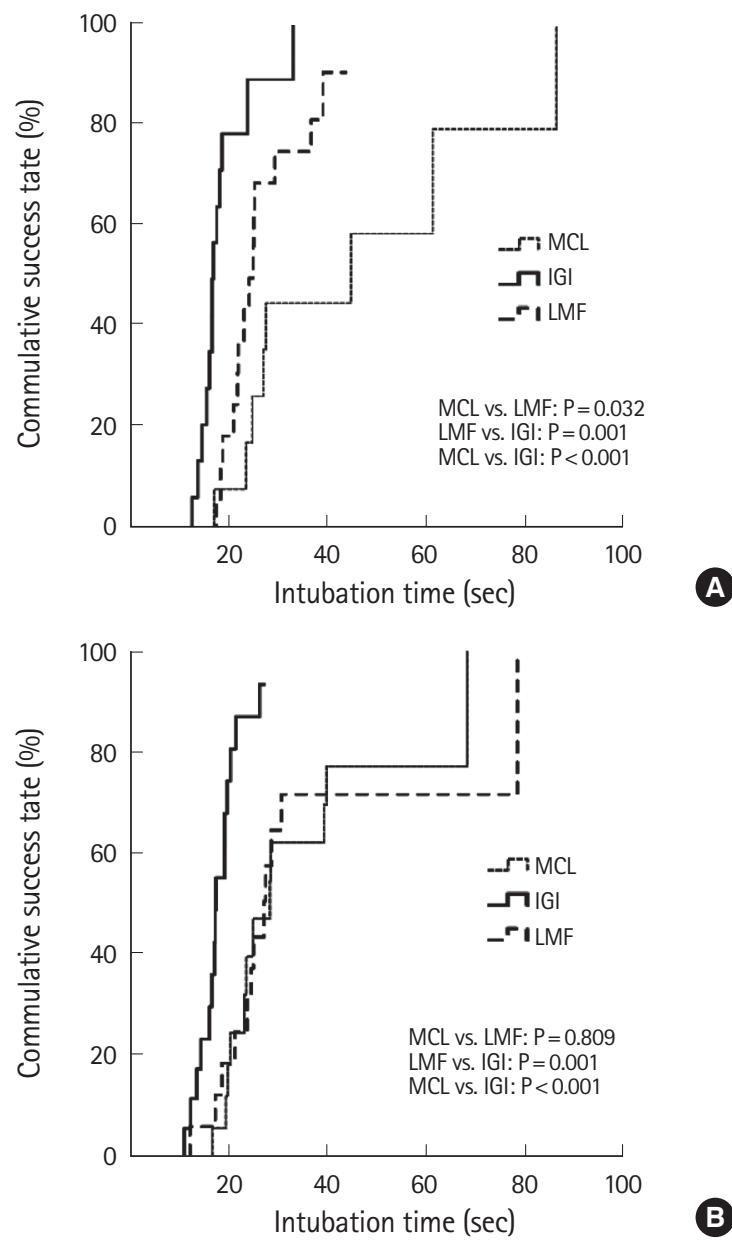

B

Fig. 2. Comparison of cumulative success rate related to total intubation time at (A) ground-level and (B) stretcher-level positions. P-value less than 0.017 is considered statistically significant. $\mathrm{MCL}$, Macintosh laryngoscope; IGI, i-gel blind intubation; LMF, laryngeal mask airway Fastrach.

(LMF vs. IGI, $\mathrm{P}<0.001 ; \mathrm{IGI}$ vs. MCL, $\mathrm{P}=0.006$ ) (Table 3 ).

The success in the IGI was significantly related to patient position among three intubation techniques $(P=0.007)$. The glottis view of MCL (Cormack and Lehane I-II) was not significantly related to patient position $(\mathrm{P}=0.79)$.

\section{Cumulative success rate at two different positions}

Regarding the cumulative success rates, IGI was the fastest among the three intubation techniques at both patient positions (all $\mathrm{P}<0.017$ ). LMF was not significantly different from $\mathrm{MCL}$ at both patient positions (in ground-level, $\mathrm{P}=0.03$; in stretcher-lev$\mathrm{el}, \mathrm{P}=0.80$ ) (Fig. 2).

\section{Factors influencing successful intubation}

The success of intubation was affected by the type of intubation technique. Although the success using LMF was equal to that of
Table 4. Factors associated with successful intubation

\begin{tabular}{llc}
\hline Successful intubation & OR $(95 \% \mathrm{Cl})$ & P-value \\
\hline Female) & $0.2(0-1.6)$ & 0.16 \\
Age $(\mathrm{yr})$ & $0.9(0.7-1.0)$ & 0.19 \\
Height $(\mathrm{cm})$ & $1.0(0.9-1.1)$ & 0.41 \\
Weight $(\mathrm{kg})$ & $0.9(0.8-1.0)$ & 0.13 \\
Paramedic experience (mon) & $1.0(0.9-1.0)$ & 0.20 \\
Intubation experience (times) $^{\mathrm{a})}$ & $0.9(0.8-1.0)$ & 0.22 \\
Ground-level $^{\mathrm{c})}$ & $0.6(0.2-1.6)$ & 0.34 \\
IGI $^{\mathrm{d})}$ & $3.6(1.1-11.6)$ & 0.03 \\
LMF $^{\mathrm{d})}$ & $2.4(0.8-7.3)$ & 0.10
\end{tabular}

$\mathrm{OR}$, odds ratio; $\mathrm{Cl}$, confidence interval; IGI, i-gel blind intubation; LMF, laryngeal mask airway Fastrach.

${ }^{a)}$ Calculated by multivariate logistic regression analysis (enter). ${ }^{b)}$ Compared to male. ${ }^{c}$ Compared to stretcher-level patient position. ${ }^{\text {d) }}$ Compared to Macintosh laryngoscope.

MCL, IGI achieved more success than MCL (odds ratio, 3.6; 95\% confidence interval, 1.1 to $11.6 ; P=0.03$ ). In contrast, the patient positions, the paramedic experience, and intubation experience were not independent factors affecting the success of intubation (Table 4).

\section{DISCUSSION}

To our knowledge, this is the first study for prehospital ground level intubation using IGI for trauma patients. In our study, IGI had a better performance compared with LMF and MCL, regardless of patient positions.

Head and neck trauma can cause airway obstruction via blood or vomitus of patients. In addition, ETI may become more difficult in cervical-immobilized patients. One previous study reported that blood or vomitus may impair visualization of the glottic inlet in out-of hospital patients when using MCL. ${ }^{5}$ Although paramedics performed VL assisted ETI, they were unable to perform ETI in $7.5 \%$ of patients, primarily because of obstructed glottic views due to secretions and blood. ${ }^{10}$ Therefore, we investigated the intubation performance of IGI and LMF as a rescue device in traumatic patients.

We compared intubation performances according to patient positions at stretcher- and ground-level. In the ground intubation using $M C L$, intubators have difficulty in laryngeal exposure. Hence, we considered stretcher-level positions similar to the sternum-level of intubators that makes an excellent view of the glottis possible." We anticipated that MCL in the stretcher-level would show a better intubation performance compared with ground intubation. However, there were no differences of the intubation time and success rate of MCL between the two patient positions, as shown in Table 2 . This result was similar to a previ- 
ous study of paramedic ETI in different patient positions. ${ }^{12}$ In obtaining a good glottic view using MCL, the ETI in the stretcherlevel was not significantly different from ground intubation.

DT of IGI was significantly shorter than that of LMF at the stretcher-level position, as shown Table 3. The shortened DT of IGI might be considerably affected by the i-gel as a non-inflationcuff device. When using IGI, intubators can save the time of inflating a cuff compared with other inflation-cuff devices, such as the LMF. Additionally, i-gel has the additional advantage of the thermoplastic elastomeric cuff design, which allows an optimal seal and reliable insertion of the i-gel. Hence, there might be some benefit for hypoxia in trauma patients from the shortened intubation time of IGI comparing with LMF. However, we thought that there was no significant difference of clinical efficacy as a rescue device for trauma patients between IGI and LMF since the success rates of both devices were similar.

Kim et al. ${ }^{9}$ reported that the significant factors affecting intubation time were intubation experience and the type of laryngoscope, not the patient position, in the comparison of VL with MCL. Similarly, our study found that the type of intubation technique significantly affected intubation success, and IGI achieved more success than MCL (Table 4). As shown in Table 1, we measured intubation performance of less experienced paramedics. If experienced paramedics are enrolled in the next study, the measured intubation performance could differ from that of this study.

There were several limitations to this study. First, we used an airway trainer manikin, reflecting a regulated simulated airway using cervical immobilization in trauma patients. However, we did not simulate the distorted laryngeal anatomy in the manikin. Hence, the measured intubation performance could differ from that of traumatic patients who have a distorted larynx. Second, we did not simulate blood or vomitus in traumatic patients. If blood or vomitus occurs in ETI using MCL, it could shorten the required time for enduring hypoxia and interfere with the laryngeal exposure. Third, regardless of 30-minute washout period for crossover, a learning effect might still remain and it could affect the intubation performance of participants in phase 2 .

In conclusion, the patient position did not affect intubation performance. Regardless of patient position, the intubation time of ETI through SADs, especially IGI, was significantly shorter than that of MCL.

\section{CONFLICT OF INTEREST}

No potential conflict of interest relevant to this article was reported.

\section{ACKNOWLEDGMENTS}

This research was supported by Ministry of Public Safety and Security (MPSS-Fire Safety-2015-76).

\section{REFERENCES}

1. Hussain $L M$, Redmond AD. Are pre-hospital deaths from accidental injury preventable? BMJ 1994;308:1077-80.

2. Khan RM, Sharma PK, Kaul N. Airway management in trauma. Indian J Anaesth 2011;55:463-9.

3. Rotondo MF, McGonigal MD, Schwab CW, Kauder DR, Hanson CW. Urgent paralysis and intubation of trauma patients: is it safe? J Trauma 1993;34:242-6.

4. Adnet F, Lapostolle F, Borron SW, Hennequin B, Leclercq $G$, Fleury M. Optimization of glottic exposure during intubation of a patient lying supine on the ground. Am J Emerg Med 1997;15:555-7.

5. Russo SG, Nickel EA, Leissner KB, Schwerdtfeger K, Bauer M, Roessler MS. Use of the GlideScope-Ranger for pre-hospital intubations by anaesthesia trained emergency physicians: an observational study. BMC Emerg Med 2016;16:8.

6. Yamada R, Maruyama K, Hirabayashi G, Koyama Y, Andoh T. Effect of head position on the success rate of blind intubation using intubating supraglottic airway devices. Am J Emerg Med 2016;34:1193-7.

7. Kim YY, Kang GH, Kim WH, et al. Comparison of blind intubation through supraglottic devices and direct laryngoscopy by novices: a simulation manikin study. Clin Exp Emerg Med 2016;3:75-80.

8. Halwagi $A E$, Massicotte $N$, Lallo $A$, et al. Tracheal intubation through the I-gel supraglottic airway versus the LMA Fastrach: a randomized controlled trial. Anesth Analg 2012;114: 152-6.

9. Kim W, Lee $Y$, Kim C, et al. Comparison of the pentax airwayscope, glidescope video laryngoscope, and macintosh laryngoscope during chest compression according to bed height. Medicine (Baltimore) 2016;95:e2631.

10. Cavus E, Callies A, Doerges V, et al. The C-MAC videolaryngoscope for prehospital emergency intubation: a prospective, multicentre, observational study. Emerg Med J 2011;28:650-3.

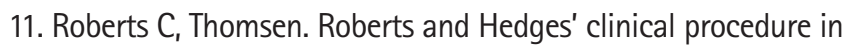
emergency medicine. 5th ed. Philadelphia, PA: Elsevier Saunders; 2009.

12. Clemency BM, Roginski M, Lindstrom HA, Billittier AJ. Paramedic intubation: patient position might matter. Prehosp Emerg Care 2014;18:239-43. 